\title{
Machine Learning Using Big Data Link Stability Based Node Observation for IoT Security
}

\author{
R.Ganesh Babu ${ }^{\text {a,1 }}$, S.Yuvaraj ${ }^{\text {a }}$, A.VedanthSrivatson ${ }^{\text {b }}$, T.Ramachandran ${ }^{\text {b }}$, \\ G.Vikram ${ }^{\mathrm{b}}$, N.Niffarudeen ${ }^{\mathrm{b}}$ \\ ${ }^{a}$ Associate Professor, Department of Electronics and Communication Engineering \\ ${ }^{a}$ Assistant Professor, Department of Electronics and Communication Engineering \\ ${ }^{b}$ UG Student, Department of Electronics and Communication Engineering \\ SRM TRP Engineering College, Tiruchirappalli, TN, India
}

\begin{abstract}
IoT systems create a multi-hop organizational structure among mobile devices in required to send on data groups. The remarkable properties of gadgets frameworks cause communications to interconnect among competing handheld devices. Most physiological directing displays don't believe secure associations all through bundle communication to organize high communicate ability and genetic blocks that also prompts increased delay as well as bundle decreasing in mastermind. Only with continued growth and transformation of IoT networks, attacks on such IoT systems are increasing at an alarming rate. Our purpose will provide researchers with a research resource on latest research patterns in IoT security. As the primary driver of with us research problem concerning IoT security as well as machine learning. This analysis of the literature among the most research literature in IoT security recognized some very key current research which will generate organizational investigations. Only with fast emergence of different IoT threats, it is essential to develop frameworks that could integrate cutting-edge big data analytics and machine learning advanced technologies. Effectiveness are critical quality variables in shaping the best methods and algorithms for detecting IoT threats in realtime or close to real time.
\end{abstract}

Keywords. Machine Learning; Physical Routing Protocol; Big Data; Traffic Density, Node Observation; Link Stability, IoT Security.

\section{Introduction}

Previous experts consider Connectivity which enable unavoidable availability among devices and do not rely on costly framework system. Communications between devices and previous establishment discharge a wide range of skilled applications besides explorers to motorists. The implementations provide security as well as reassurance to drivers by allowing them to collaborate and communicate with each other in order to avoid any mishaps, for example, a road transformed parking garage, innocuous barriers, speed violation, access, temperature records, sight as well as sound congresses, and etc.

\footnotetext{
${ }^{1}$ R.Ganesh Babu, Associate Professor, Department of Electronics and Communication Engineering, SRM TRP Engineering College, Tiruchirappalli, TN, India; Email: ganeshbaburajendran@gmail.com.
} 
Regardless of the fact it is a subclass of compact specially designated systems, a network device does have few distinguishing characteristics that distinguish from other improvised systems [1]. The much more important distinctions are the elevated simplicity schedule; rapidly constantly evolving configuration results in higher framework fragmentation and dysconnectivity in sorting out. Amazing optimization techniques, in any scenario, are also not completely sporadic, and development of sensor hubs is constrained by ways as well as, in the most portions, evident.

The Internet of Things (IoT) is just a network with billions of connected devices which can send or receive information over the network. Nowadays, these systems could be located in a variety of settings, including homes, workplaces, transportation, medicare, telecommunications, agriculture, and so on. IoT systems are rapidly expanding, making a significant difference with us everyday lives and assisting industries such as healthcare as well as transport infrastructure in making decisions. According to the research results of Business Analyst's 2020 IoT document, the IoT size is forecast to grow by more than $\$ 2.5$ trillion per year by 2028 . This contains an increase in the number of IoT gadgets from 9 billion in 2019 to 44 billion besides 2028 . IoT has decided to bring substantial benefits to everyone lives, social system, and industrial sectors over its years; that being said, its technology was using has yet to mature sufficiently to provide utilized to carry as well as communication. As the number of devices connected grows, adversaries have more opportunities to gain direct exposure to all of them and are using them to release huge threats.

Securing IoT gadgets is becoming increasingly difficult for both producers and consumers. Several of the significant security challenges recognized mostly by researchers include weak, fallback, or internet data storage without even a passcode. IoT gadgets are frequently shipped to switch, simple, or no passcode. Hackers can very easily exploit such vulnerability by gaining access to these devices. Such vulnerability puts the consumers' privacy at risk and allows hackers to use IoT devices to launch large-scale attacks like DDoS. It confirmed that even an unclassified health history of

over 6 million people in the United States, or even huge sizes globally, is accessible online. This data is kept online from over 197 servers, because anyone with an internet browser can availability it by operating effective governance. According the Sophistry document [8], the ten largest credit and quickly guessable passcode being used with IoT gadgets attacks are as follows are shown in Figure 1.

Only with help of machine learning (ML) and thoughtful threats in IoT gadgets, it is essential to identify a defense actions and understand important limitations within the security measures once more for interchange in diverse networks[9]. This procedure is challenging since an IoT device with limited abilities frequently has complexity correctly forecasting the whole infrastructure as well as inflict damage level[10].

\section{Machine learning in IoT Security}

Throughout this we will discuss the inspiration for using ML throughout IoT security inside the context of existing information security used within IoT networks. We begin by shedding light also on particular qualities of IoT networks and is relevant with security, but instead we start debating the security threats that impede IoT implementation, and also the holes in current security products. After with the intention of establish the incentive for utilize ML to deal with security deal with in IoT gadgets[1]. 


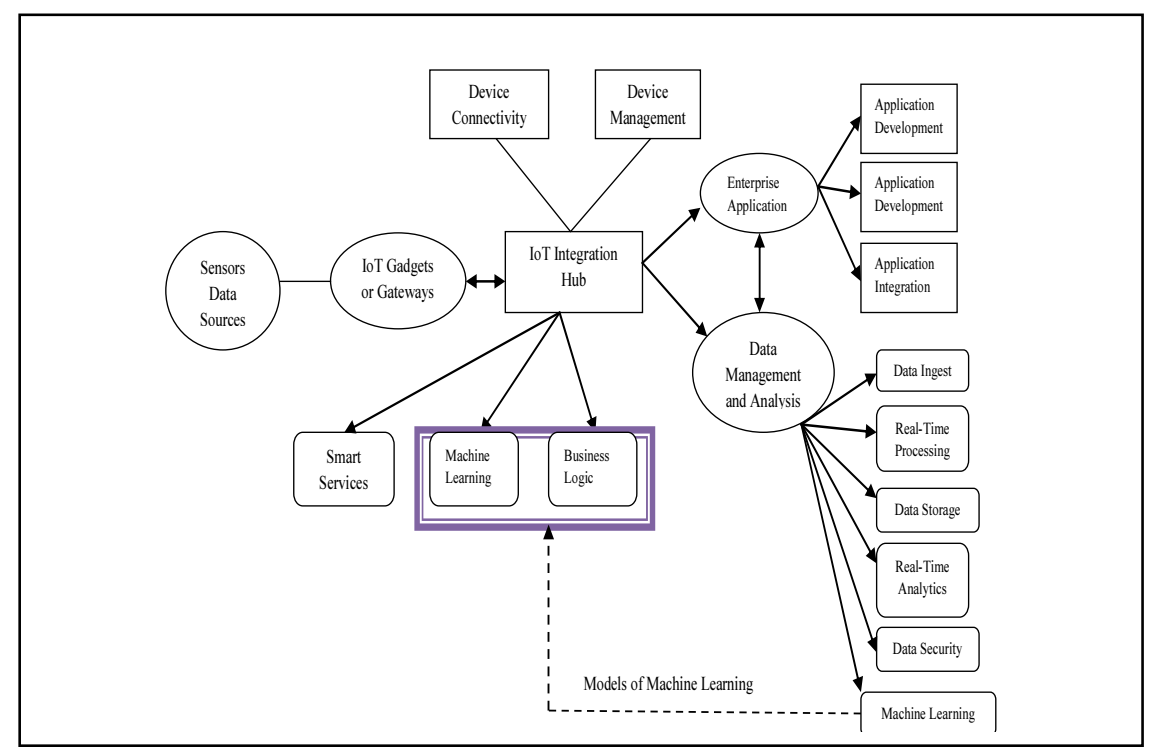

Figure 1. Machine Learning with IoT Security using Big Data Analysis

\section{Security Challenges in IoT Deployment}

Data protections are two of the most popular considerations with in commercialization of IoT applications and services. The host Server is an enticing playground besides security threats starting from pure bots to organizational excellently data breaches which have harmed multiple sectors such as wellbeing as well as corporate [2]. The restrictions of IoT gadgets, as well as the environment of the organization, present unique challenges for the security of the both gadgets and applications. To date, security requirements in the IoT system are being thoroughly studied from different perspectives, including secure communication, information security, confidentiality, architecture and design security, information security, threat intelligence, and etc [3]. It concentrated on the differences and similarities between IoT as well as conventional IT gadgets in terms of security. They as well concentrated on privacy concerns. The main motivators for arguing about commonalities and contrasts are technology, equipment, networks, and implementations.

\section{Machine Learning and IoT Security}

Throughout this segment, we will look at machine learning techniques and how they can be used in IoT systems.

$$
\text { Dis } \tan c e=\sqrt{\left(X_{2}-X_{1}\right)^{2}+\left(Y_{2}-Y_{1}\right)^{2}}
$$

There are four types of ML algorithms: monitored, unmonitored, semi-supervised, as well as validation classification techniques. 


$$
M_{s}=M_{1}+M_{2}
$$

Where

$$
M_{1}=\operatorname{Radius}_{1}^{2} \cdot a_{2}-\frac{\text { Radius }_{1}^{2} \cdot \sin \left(2 a_{1}\right)}{2}
$$

$$
B_{2}=\text { Radius }_{1}^{2} \cdot \alpha_{2}-\frac{\text { Radius }_{1}^{2} \cdot \sin \left(2 \alpha_{1}\right)}{2}
$$

The line connecting the source as well as impartial center points $90^{\circ}=\left(\alpha_{1}=45^{\circ}\right)$ is a point bisector; throughout this method, the half-region is used $M_{S}$.

$$
A=\operatorname{Radius}_{1}^{2}\left[\frac{\pi-2}{4}\right]+\operatorname{Raduis}_{2}^{2}\left[\alpha_{2}-\frac{\sin \left(2 \alpha_{1}\right)}{2}\right]
$$

Supervised Classification: It is occurs while potential measures have been delineated to be attained from a number of objects [4]. For such a method of training, its data is classified initially, then trained with the classification model.

$$
D i s_{w v}=\max \left[\log \left[\frac{C D_{s, d}}{C D_{j, d}}\right], 0.1\right]
$$

Unmonitored Learning: It is the climate only significantly contributes without any desired outcomes. It's doesn't require classification model and can analyze similarities among unstructured data as well as classify it into various groups.

$$
D i r_{w v}=\left[L Q\left(\vec{D}_{n}, \vec{D}_{p t}\right)\right]
$$

Reinforcement Learning (RL): No desired objectives are described in Reinforcement Learning (RL), as well as the representative discovers from responses after observing the environment.

$$
T D_{w v}=\left[1-D_{c}\right]+\left[\min \left[\frac{N_{a v g}}{N_{c o n}}, 1\right]\right]
$$

It's also very critical to select the appropriate objective functions as its agent's future direction is determined by the reward systems accumulated [5]. With in opposite situations, RL technologies are frequently used:

- When there is a lack of critical data and previous examples besides training phase.

- The precise correct and incorrect values again for particular circumstance are unknown a priori. 
- The ultimate goal is recognized, and the climate could be discerned in order to increase both short-term as well as long-term benefits.

\section{Function of Basic ML Techniques}

Monitored and unmonitored learning techniques are primarily used to solve predictive analytics problems, whereas RL is used to solve comparative analysis as well as decision-making problems [6]. The nature of the data available influences this classification and the decision of ML Classification algorithms. Classification model is used when its category of input data as well as the target output (labels) are recognized [8] . In this case, the system has only been given training to information of the user to expected output. Learning algorithms are two classifier techniques; to regression dealing with constant outputs as well as classification dealing with separable outputs is show in Figure 2. Support Vector Regression (SVR), regression methods, and memory modules are examples of frequently utilized logistic regression.

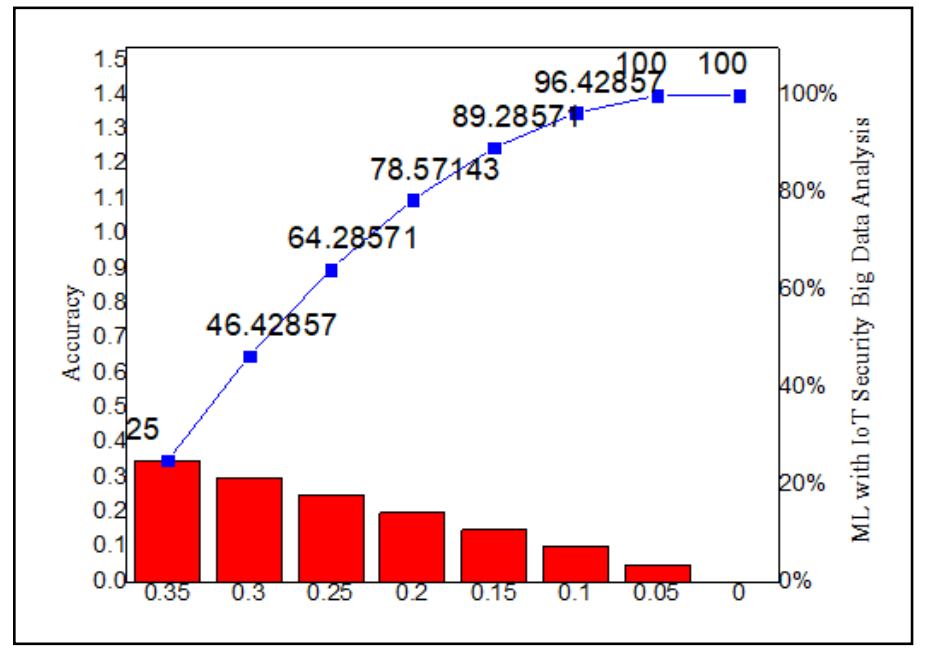

Figure 2. Big Data Analysis for Machine Learning with IoT Security

Classification, from the other side, works to discontinuous target value [7-9]. Classification algorithms that are commonly include using K-nearest neighbour, regression analysis, as well as Support Vector Machine (SVM). Several other algorithms, including such neural networks, are used for classification as well as reversion [10]. Supervised classification techniques can be used to classify the device when the deliverables weren't very well but the system must uncover the framework inside the original data. Clustering, that also group's items following established number of clusters including such K-means cluster analysis, is show in Figure 3.

\section{Conclusion}

Despite the fact that with us concentrate in this systematic review (SLR) had been on a quite short period of time, the comparatively lot of research articles published indicated this is a popular area of research. This area of research combines several fast- 
increasing fields: IoT, protection, machine learning, as well as data analytics are all buzzwords these days. We chose mentioned research information to check our SLR in order to assist researchers in identifying current trends inside the chosen research area. We encapsulated specific topics including such growing in popularity.

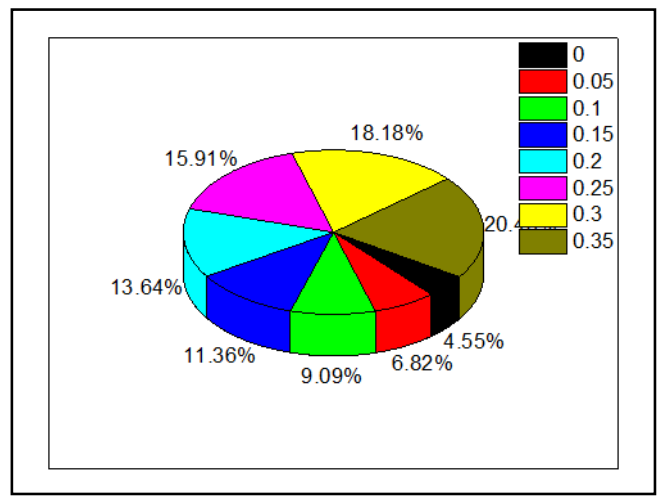

Figure 3. Performance Analysis for Machine Learning Techniques using Big Data link stability in Node Observation for IoT Security

\section{References}

[1] Hossain, E., Khan, I., Un-Noor, F., Sikander, S. S., \& Sunny, M. S. H. (2019). Application of Big Data and Machine Learning in Smart Grid, and Associated Security Concerns: A Review. IEEE Access, 7, 13960-13988. https://doi.org/10.1109/ACCESS.2019.2894819

[2] Karthika, P., Ganesh Babu, R., \& Karthik, P. A. (2020). Fog Computing using Interoperability and IoT Security Issues in Health Care (pp. 97-105). https://doi.org/10.1007/978-981-15-2329-8_10

[3] Babu, R. G., Nathan, V. V., Bino, J., Amali, C., \& Ganesh, S. (2021). IoT Security Enhancement with Automated Identification Device using IOT SENTINEL. 2021 11th International Conference on Cloud Computing, Data Science \& Engineering (Confluence), 518-523. https://doi.org/10.1109/ Confluence51648.2021.9377165

[4] C., C., R., G. B., M., S., M., A., R., B., \& Balaji, M. (2020). Machine Learning Based Condition Recognition System for Bikers. 2020 7th International Conference on Smart Structures and Systems (ICSSS), 1-6. https://doi.org/10.1109/ICSSS49621.2020.9202245

[5] Babu, R. G., Nedumaran, A., \& Sisay, A. (2019). Machine Learning in IoT Security Performance Analysis of Outage Probability of link selection for Cognitive Networks. 2019 Third International Conference on I-SMAC (IoT in Social, Mobile, Analytics and Cloud) (I-SMAC), 15-19. https://doi.org/10.1109/I-SMAC47947.2019.9032669

[6] Karthika, P., Babu, R. G., \& Nedumaran, A. (2019). Machine Learning Security Allocation in IoT. 2019 International Conference on Intelligent Computing and Control Systems (ICCS), 474-478. https://doi.org/10.1109/ICCS45141.2019.9065886

[7] Ali, E. S., Hasan, M. K., Hassan, R., Saeed, R. A., Hassan, M. B., Islam, S., Nafi, N. S., \& Bevinakoppa, S. (2021). Machine Learning Technologies for Secure Vehicular Communication in Internet of Vehicles: Recent Advances and Applications. Security and Communication Networks, 2021, 1-23. https://doi.org/10.1155/2021/8868355

[8] Soe, Y. N., Feng, Y., Santosa, P. I., Hartanto, R., \& Sakurai, K. (2020). Machine Learning-Based IoTBotnet Attack Detection with Sequential Architecture. Sensors, 20(16), 4372. https://doi.org/10.3390/s20164372

[9] Syafrudin, M., Alfian, G., Fitriyani, N., \& Rhee, J. (2018). Performance Analysis of IoT-Based Sensor, Big Data Processing, and Machine Learning Model for Real-Time Monitoring System in Automotive Manufacturing. Sensors, 18(9), 2946. https://doi.org/10.3390/s18092946 
[10] Rehman, A., Haseeb, K., Saba, T., Lloret, J., \& Tariq, U. (2021). Secured Big Data Analytics for Decision-Oriented Medical System Using Internet of Things. Electronics, 10(11), 1273. https://doi.org/10.3390/electronics10111273 